\title{
Zinc fingers as protein recognition motifs: Structural basis for the GATA-1/Friend of GATA interaction
}

\author{
Chu Kong Liew*, Raina J. Y. Simpson*, Ann H. Y. Kwan*, Linda A. Crofts, Fionna E. Loughlin, Jacqueline M. Matthews, \\ Merlin Crossley, and Joel P. Mackay ${ }^{\dagger}$
}

School of Molecular and Microbial Biosciences, University of Sydney, Sydney, New South Wales 2006, Australia

Edited by Stuart H. Orkin, Harvard Medical School, Boston, MA, and approved December 8, 2004 (received for review October 11, 2004)

\begin{abstract}
GATA-1 and friend of GATA (FOG) are zinc-finger transcription factors that physically interact to play essential roles in erythroid and megakaryocytic development. Several naturally occurring mutations in the GATA-1 gene that alter the FOG-binding domain have been reported. The mutations are associated with familial anemias and thrombocytopenias of differing severity. To elucidate the molecular basis for the GATA-1/FOG interaction, we have determined the three-dimensional structure of a complex comprising the interaction domains of these proteins. The structure reveals how zinc fingers can act as protein recognition motifs. Details of the architecture of the contact domains and their physical properties provide a molecular explanation for how the GATA-1 mutations contribute to distinct but related genetic diseases.
\end{abstract}

transcription | factor | gene expression

$T^{*}$ he erythroid transcription factor GATA-1 has become a paradigm for understanding the control of gene expression by sequence-specific DNA-binding proteins. Numerous studies (reviewed in refs. 1 and 2) have revealed that the function of GATA-1 depends on its ability to bind both DNA and other proteins such as the DNA-binding proteins PU.1, Fli-1 Sp1, and erythroid Krüppel-like factor, as well as coregulators such as LIM-only 2 protein, cyclic AMP response element-binding protein-binding protein, and the zinc-finger protein friend of GATA (FOG). It is thought that different partnerships involving GATA-1 occur at different DNA elements, in different cell types, and at distinct stages of development, and that these regulatory networks contribute to the ability of GATA-1 to direct the complex patterns of gene expression required for the differentiation of blood cells.

GATA-family proteins contain two highly conserved trebleclef zinc fingers ( $\mathrm{ZnFs}$ ) domains (3): the N-terminal (NF) and the C-terminal (CF) fingers. These domains mediate both DNA and protein interactions, but despite high sequence similarity to each other ( $>50 \%$ identity), have quite distinct functions. The $\mathrm{CF}$ appears to be the main determinant of DNA-binding and recognizes sequences of the form $\mathrm{T} / \mathrm{AGATAA} / \mathrm{G}$, whereas the $\mathrm{NF}$ can participate in binding to tandem T/AGATAA/G sites (ref. 4 and references therein) and can independently bind GATC motifs (5). The protein-binding specificities of the two ZnFs also differ; most notably, the NF alone is able to recruit FOG, an $\approx 1,000$-aa protein (6) that contains five classic CysCys-His-His $\left(\mathrm{C}_{2} \mathrm{H}_{2}\right)$ and four related Cys-Cys-His-Cys $\left(\mathrm{C}_{2} \mathrm{HC}\right)$ ZnFs (Fig. 1A).

FOG is essential for the normal development of both erythrocytes and megakaryocytes (7) and the interaction of FOG with GATA-1 is indispensable for these events (8). Of the nine $\mathrm{ZnFs}$ in FOG (Fig. 1A), four are not found in the tandem arrays that are generally associated with DNA-binding activity. Intriguingly, each of these four isolated domains can independently bind NF, consistent with the emerging view that single fingers can mediate protein-protein interactions $(9,10)$.

Here, we describe the solution structure of the protein-protein complex formed by the interaction domains of GATA-1 and FOG. The structure reveals for the first time how $\mathrm{ZnF}$ domains can act as protein recognition motifs. We also show that NF can bind simultaneously both to FOG and to DNA, demonstrating that even domains of only $\approx 40$ aa can harbor two completely distinct recognition surfaces. Finally, we define the molecular causes for several human blood disorders that arise from mutations in GATA-1.

\section{Materials and Methods}

Sample Preparation. The GATA-1 NF and dFOG finger 1 were overexpressed and purified as described (11). NF mutants were purified either in the same fashion or by size exclusion chromatography. Complexes were prepared by mixing stoichiometric quantities of each domain dissolved in $20 \mathrm{mM}$ sodium acetate ( $\mathrm{pH}$ 5.5). In each complex, one of the two components was uniformly ${ }^{15} \mathrm{~N},{ }^{13} \mathrm{C}$-labeled, whereas the other was unlabeled. Oligonucleotides (GCAACTGATCTGGACT and its complement) used for isothermal titration calorimetry were purchased from Sigma in the trityl-on form, simultaneously deprotected and purified by reverse-phase HPLC, annealed, and then purified on a Superdex 75 column.

NMR Spectroscopy. NMR data were acquired on a Bruker 600$\mathrm{MHz}$ spectrometer. All experiments were carried out at $280 \mathrm{~K}$ at concentrations of 400-600 $\mu \mathrm{M}$. Spectra were analyzed by using XEASY (12). Backbone and side chain resonance assignments were obtained by using standard triple resonance experiments (13), namely HNCA, HN(CO)CA, HNCACB, CBCA(CO)NH, HCCH-TOCSY, HCCH-COSY, and HNCO. Chemical shift indices were calculated as described (14). Torsion angle restraints were obtained from HNHA experiments (15) and TALOS (16). Intermolecular distance constraints were obtained by using $2 \mathrm{D}$ NOESY and $2 \mathrm{D}{ }^{12} \mathrm{C}$-filtered $/{ }^{13} \mathrm{C}$-edited and ${ }^{15} \mathrm{~N}$ filtered $/{ }^{13} \mathrm{C}$-edited NOESY experiments (mixing time, $100 \mathrm{~ms}$ ) (17); all constraints were set to upper distance limits of $6 \AA$.

Structure Determination and Analysis. Preliminary structure calculations were performed in DYANA $(18)$ and later in ARIA $(19,20)$. Zinc coordination was incorporated in the ARIA calculations (21). HADDOCK (22) was used to dock mNF and dFOG-F1, using the previously determined structures of both domains $(11,23)$ and 35 intermolecular NOEs derived from analysis of NOE data. Ambiguous restraints (15) based on chemical shift perturbation studies were also added, but did not significantly improve the quality of the structures. A total of 500 rigid body docking trials were carried out by using the standard HADDOCK protocol, with the 100 lowest energy solutions used for subsequent semiflexible

This paper was submitted directly (Track II) to the PNAS office.

Abbreviations: FOG, friend of GATA; ZnF, zinc finger; NF, N-terminal ZnF; CF, C-terminal ZnF.

Data deposition: The atomic coordinates and structure factors have been deposited in the Protein Data Bank, www.pdb.org (PDB ID code 1YOJ).

*C.K.L., R.J.Y.S., and A.H.Y.K. contributed equally to this work.

†To whom correspondence should be addressed. E-mail: j.mackay@mmb.usyd.edu.au.

() 2005 by The National Academy of Sciences of the USA 


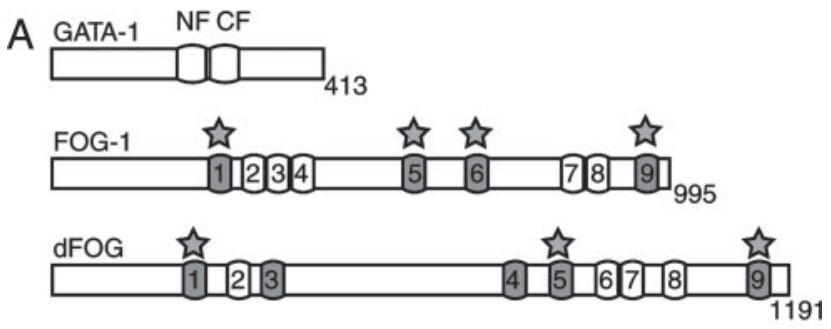

B
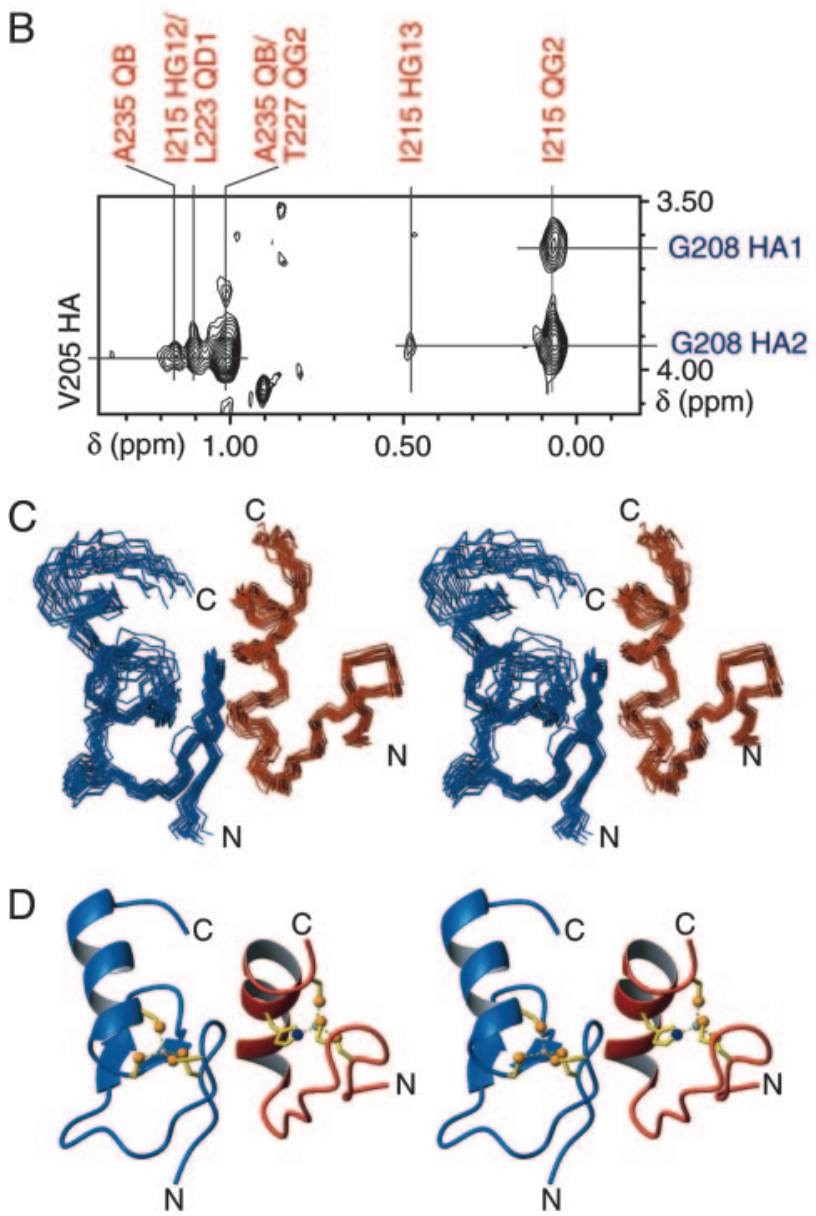

Fig. 1. Structure of the GATA/FOG complex. (A) Domain structures of GATA-1, FOG-1, and Drosophila FOG (U-shaped). NF, N-terminal ZnF; CF, C-terminal ZnF. Shaded ZnFs have a CCHC topology, and domains marked with a star can bind NF. Amino acid numbers are also given. $(B)$ Section of a 2D $\mathrm{F}_{1}-{ }^{13} \mathrm{C}$-filtered, $\mathrm{F}_{2}{ }^{13} \mathrm{C}$-edited NOESY of the $\left[{ }^{15} \mathrm{~N},{ }^{13} \mathrm{C}\right] \mathrm{dFOG}-\mathrm{F} 1 / \mathrm{mNF}$ complex. Intermolecular NOEs between protons from dFOG-F1 (red) and mNF (blue) are shown. Numbering is for the full-length proteins. (C) Stereo diagram of the overlay of the top 20 structures of the GATA-1/FOG complex. mNF is shown in blue and dFOG-F1 is shown in red. $(D)$ Ribbon diagram of the lowest energy structure of the complex. Zinc ions are shown in gray and zinc-ligating side chains are shown in gold and blue.

simulated annealing and water refinement. The 20 with the lowest energy were taken to represent the structure of the complex. The structures were analyzed by using PROCHECK (24) and LIGPLOT (25).

Isothermal Titration Calorimetry. NF/DNA titrations were performed at $298 \mathrm{~K}$ in $10 \mathrm{mM}$ sodium phosphate $/ 0-10 \mathrm{mM}$ $\mathrm{NaCl} / 0.5-1 \mathrm{mM}$ DTT/0.1-0.3 mM ZnSO 4 , pH 6.5-7.5. NF (190 $\mu \mathrm{M}), \mathrm{V} 205 \mathrm{M}(220 \mu \mathrm{M}), \mathrm{G} 208 \mathrm{~S}(50 \mu \mathrm{M})$, R216Q (45 $\mu \mathrm{M})$, and D218G $(140 \mu \mathrm{M})$ were titrated into a solution containing the double-stranded 16-bp oligonucleotide $(3-10 \mu \mathrm{M})$. NF/FOG titrations (using dFOG-F1) were performed in $20 \mathrm{mM}$ sodium acetate $/ 1.5 \mathrm{mM} \mathrm{ZnSO}_{4} / 0.1 \mathrm{mM}$ (2-carboxyethyl)phosphine hydrochloride (TCEP) at $\mathrm{pH} 5.0$. NF $(20 \mu \mathrm{M})$, V205M $(25 \mu \mathrm{M})$, G208S $(20 \mu \mathrm{M})$, R216Q $(40 \mu \mathrm{M})$, and D218G $(50 \mu \mathrm{M})$ were titrated with dFOG-F1 $(305,435,305,670$, and $670 \mu \mathrm{M}$, respectively). Previous work established that the affinity of this interaction is unchanged between $\mathrm{pH} 5$ and 7 (26).

Interaction Assays. Biotin-labeled sense and unlabelled antisense oligonucleotides (200 pmol/mg beads), encompassing the GATA binding site in the mouse $\alpha$ globin proximal promoter (m1a-GATA: GAT CTC CGG CAA CTG ATA AGG ATT CCC), a GATC site from the 5-aminolevulinate synthase proximal promoter (ALAS2: AAG GAT GGT CTG ATC TCA AAA TCG AA), or GATA $\rightarrow$ CTTA mutants of these sites, were annealed and coupled to Dynabeads M-280 Streptavidin (Dynal) according to the manufacturers instructions.

For pull-down assays, mouse erythroleukemia (MEL) cell nuclear extract $(250 \mu \mathrm{g})$ supplemented with nuclear extract from COS cells overexpressing FOG1 (2 $\mu \mathrm{g}$; doubling the amount of FOG present in the reaction) was incubated with $125 \mu \mathrm{g}$ of oligonucleotide-coupled beads in $500 \mu \mathrm{l}$ of binding buffer $(20$ $\mathrm{mM}$ Tris $\cdot \mathrm{Cl}$, pH 7.9 at $4^{\circ} \mathrm{C} / 5 \mathrm{mM}$ EDTA/50 mM KCl$/ 2.5 \mathrm{mM}$ $\mathrm{MgCl}_{2} / 0.05 \%$ Nonidet P-40/10\% glycerol/1 mM DTT) with protease inhibitors for $3 \mathrm{~h}$ at $4^{\circ} \mathrm{C}$. After extensive washing, bound proteins were run on SDS/PAGE and detected by Western blotting with either $\alpha$ FOG- 1 rabbit serum or $\alpha$ GATA- 1 monoclonal antibody (Santa Cruz Biotechnology). Additionally, MBP-NF and GST-FOG-finger-6 were overexpressed in Escherichia coli and purified by amylose and glutathione affinity chromatography, respectively. A mixture of the two domains $(0.5-1 \mu \mathrm{g}$ of each) was incubated with the oligonucleotidecoupled beads as described above. Proteins were detected by Western blotting with anti-MBP or anti-GST.

Electrophoretic Mobility-Shift Assays (EMSAs). GST-fusion proteins were eluted from glutathione-agarose beads with reduced glutathione $(10 \mathrm{mM})$ in $50 \mathrm{mM}$ Tris, $\mathrm{pH} 8.0 / 100 \mathrm{mM} \mathrm{NaCl} / 10 \%$ glycerol. EMSA reactions were set up in a total of $30 \mu \mathrm{l}$, comprising $\approx 5 \mu \mathrm{g}$ of GST-fusion proteins/0.8 of ng ${ }^{32} \mathrm{P}$-labeled probe $/ 10 \mathrm{mM}$ Hepes, $\mathrm{pH} 7.9 / 50 \mathrm{mM} \mathrm{KCl} / 5 \mathrm{mM} \mathrm{MgCl} / 2 / 1 \mathrm{mM}$ EDTA $/ 5 \%$ glycerol $/ 0.3 \mathrm{mM}$ DTT $/ 30 \mu \mathrm{g} / \mathrm{ml} \mathrm{BSA} / 30 \mu \mathrm{g} / \mathrm{ml}$ poly $(\mathrm{dIdC}) / 10 \mu \mathrm{M} \mathrm{ZnSO}_{4}$. The reactions were kept on ice for $15 \mathrm{~min}$ and then loaded onto a $6 \%$ native polyacrylamide gel made up in $0.5 \times$ Tris borate-EDTA. The gel was subjected to electrophoresis at $250 \mathrm{~V}$ for $90 \mathrm{~min}$, dried, and analyzed by using a PhosphorImager (Molecular Dynamics). End-labeled probes (5) included a GATC site from the ALAS2 promoter and two sequences that lacked GATC motifs: CCG CCA GGT GCT CG (probe 1) and CTA TCA GCT GAT GA (probe 2).

\section{Results}

Structure Determination. Preliminary studies revealed that the four GATA-interacting ZnFs of murine FOG were unsuitable for structural studies (27). We therefore turned to the Drosophila FOG-family protein U-shaped (dFOG; Fig. 1). This protein interacts with the GATA factors Pannier and Serpent to control development in the fly $(28-30)$. The NF of Pannier is $87 \%$ identical to murine $\mathrm{NF}$ ( $\mathrm{mNF}$ ), and we have previously shown that $\mathrm{mNF}$ can interact with fingers 1,5 , and 9 from dFOG (31). Furthermore, the complex formed between dFOG-F1 and mGATA-1 NF has an association constant that is indistinguishable from the complex formed with the Pannier NF (26).

Therefore, we determined the solution structure of the complex formed between $\mathrm{mNF}$ and $\mathrm{dFOG}-\mathrm{F} 1$ by using restraints derived from multidimensional NMR spectroscopy. Because the relatively weak association constant $\left(\approx 1 \times 10^{5} \mathrm{M}^{-1}\right)$ gave rise to exchange kinetics that broadened many resonances in the spec- 


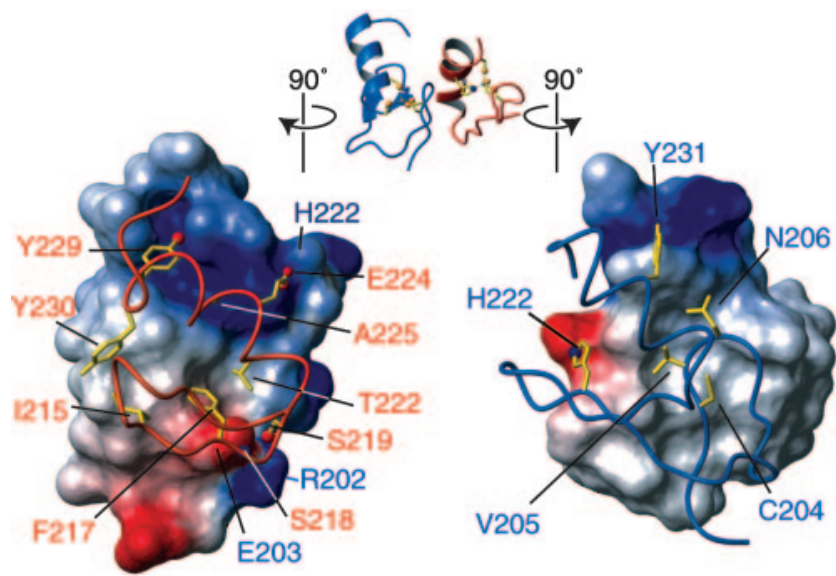

Fig. 2. The GATA-1:dFOG interface. Electrostatic surfaces of mNF (Left) and dFOG-F1 (Right) are shown (blue for mNF and red for dFOG-F1). The relative orientations of each image compared with Fig. 1 are indicated.

tra, we tried many sets of solution conditions to maximize the quality of our spectra. Once optimal conditions were established, a combination of two-dimensional double-half-filtered NOESY (17) and normal homonuclear NOESY spectra yielded a total of 35 intermolecular NOEs (Fig. $1 B$ ), as well as many intramolecular NOEs. The total number of NOEs observed was still less than expected for a complex of this size, and it was clear from examination of the spectra that exchange broadening still affected a number of resonances.

Preliminary structure calculations based on the intramolecular NOE data and TALOS (16) data established that the backbone folds of both $\mathrm{mNF}$ and dFOG-F1 are not substantially altered by formation of the GATA/FOG complex. This conclusion was confirmed by a comparison of the $\mathrm{C}^{\alpha}$ and $\mathrm{C}^{\beta}$ chemical shift indices (14) for mNF and dFOG-F1 in both the free and complexed states (see supporting information, which is published on the PNAS web site). Therefore, we used a combination of intermolecular NOEs and chemical shift perturbation data as input into HADDOCK (22) to calculate the solution structure of the complex. The structures were compared before and after annealing in water; no significant changes in the arrangement of the complex were observed.

Structure of the Complex. Fig. $1 C$ and $D$ shows the structure of the GATA-1/dFOG complex generated from HADDOCK calculations (structural statistics are provided in supporting information; Protein Data Bank code $1 \mathrm{Y} 0 \mathrm{~J}$ ). $\mathrm{mNF}$ is a treble-clef type $\mathrm{ZnF}$ comprising two irregular $\beta$-hairpins followed by a $\mathrm{C}$ terminal $\alpha$-helix (23), whereas dFOG-F1 belongs to the $\mathrm{CCHC}$ subclass of classical ZnFs (27). This domain consists of an $\mathrm{N}$-terminal $\beta$-hairpin and an $\alpha$-helix. It is the first $\beta$-hairpin, and to a lesser extent the second hairpin, of NF that contacts FOG. In contrast, the GATA-binding surface of FOG is centered primarily on the $\alpha$-helix, with some contribution from residues immediately preceding the helix. In the structure of the complex, each of the two $\mathrm{ZnFs}$ is reasonably well defined, and the backbone rms deviation (rmsd) for all structured residues in the complex is $0.79 \AA$. The water-refined structures displayed an rmsd over the same atoms of $1.0 \AA$. The complex buries $\approx 1,000$ $\AA^{2}$ of surface area, which is relatively low for a protein-protein complex (32), but reasonable given the moderate affinity of the interaction (26). ${ }^{15} \mathrm{~N}$ relaxation measurements carried out for each domain in both the uncomplexed and complexed forms (data not shown) revealed that no substantial changes in mobility take place for either domain upon complex formation. Thus,
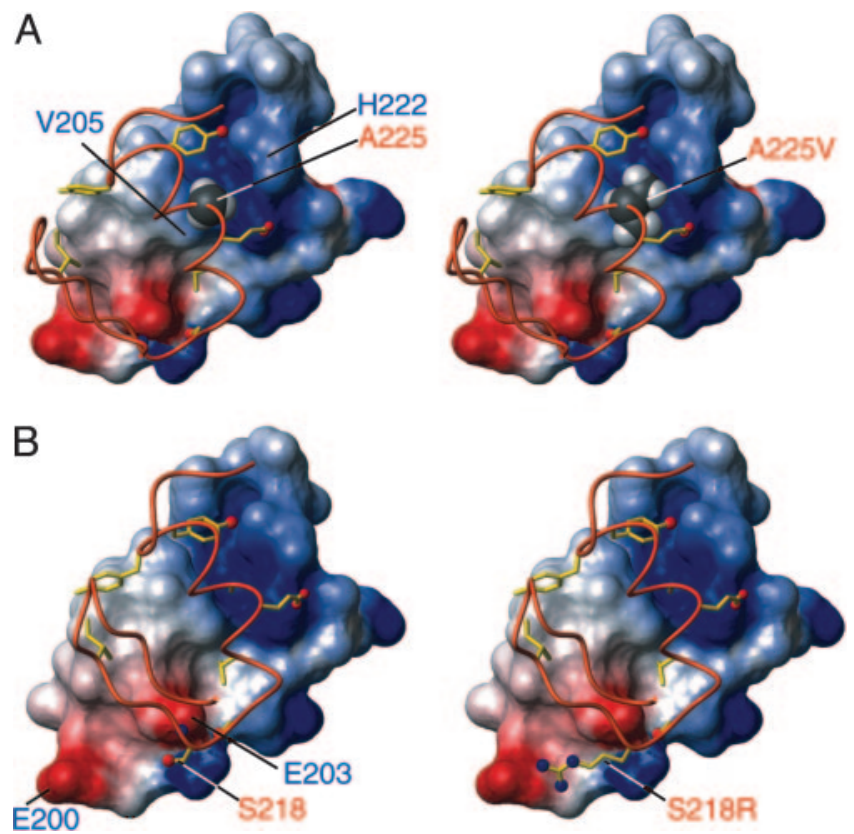

Fig. 3. Sequence diversity in GATA-binding FOG ZnFs. (A) The GATA-FOG structure showing the position of $A 225_{\mathrm{dFOG}}$ (Left), and the likely position of the valine side chain that replaces $A 225$ in a complex formed between NF and mFOG-F6 (Right). (B) Position of the S218 side chain and the two nearby glutamates from NF (Left). (Right) The likely position of the arginine side chain that would replace S218 in a NF/mFOG-F1 complex.

both domains take up relatively rigid conformations in the free and complexed states.

The two protein domains contact each other through a combination of polar and hydrophobic interactions (Fig. 2). The FOG-binding surface of $\mathrm{mNF}$ is centered on a single valine V $205_{\mathrm{NF}}$, which packs into a shallow depression on the surface of FOG (Fig. 2 Right). This interaction displays good shape complementarity, and $\mathrm{V} 205_{\mathrm{NF}}$ forms extensive hydrophobic contacts with T222 $2_{\mathrm{dFOG}}, \mathrm{A} 225_{\mathrm{dFOG}}, \mathrm{Y} 229_{\mathrm{dFOG}}$, and $\mathrm{Y} 230_{\mathrm{dFOG}}$. Y231 $1_{\mathrm{NF}}$ also contacts $\mathrm{Y} 230_{\mathrm{dFOG}}$. Similarly, the aliphatic portion of the E $203_{\mathrm{NF}}$ side chain contacts $\mathrm{F} 217_{\mathrm{dFOG}}$, and $\mathrm{H} 222_{\mathrm{NF}}$ contacts A $225_{\mathrm{dFOG}}$. The remainder of the interactions are largely polar in nature. The carboxylate side chain of E203 $3_{\mathrm{NF}}$ forms interactions with both the side chain hydroxyl of $\mathrm{S} 219_{\mathrm{dFOG}}$ and the main chain $\mathrm{H}^{\mathrm{N}}$ of $\mathrm{S} 218_{\mathrm{dFOG}}$, and the guanidine group of R202 $2_{\mathrm{NF}}$ also lies adjacent to the $\mathrm{S} 219_{\mathrm{dFOG}}$ hydroxyl. The side chain of $\mathrm{H} 222_{\mathrm{NF}}$ also pairs with $\mathrm{E} 224_{\mathrm{dFOG}}$, and the amide side chain group of N206 $\mathrm{NF}$

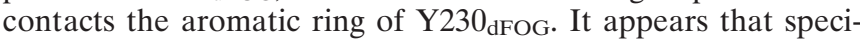
ficity is brought about through a combination of electrostatic interactions and the van der Waals contacts made by residues such as V205 $5_{\mathrm{NF}}$ upon burial from solvent water.

Sequence Conservation in the GATA/FOG Interaction. At least 11 separate $\mathrm{ZnFs}$ from FOG-1, FOG-2, and dFOG are each capable of binding to GATA-1 (31). The structure of the NF/dFOG-F1 complex allows us to understand both similarities and differences between the sequences of these ZnFs. For example, A225 $\mathrm{dFOG}$ lies at the interface with NF, contacting the side chain of V205 $\mathrm{NF}$. However, examination of the structure in this region (Fig. $3 \mathrm{~A}$ Left) reveals that a cavity exists in the region of $\mathrm{A} 225_{\mathrm{dFOG}}$. Several other GATA-interacting FOG fingers have a valine in this position, and in silico substitution of valine for alanine (Fig. 3 A Right) indicates that the valine can easily be accommodated, making additional contacts with both $\mathrm{V} 205_{\mathrm{NF}}$ and $\mathrm{H} 222_{\mathrm{NF}}$. It is notable that finger 6 of both FOG-1 and FOG-2, which are thought to be the most important ZnFs for GATA-FOG synergy, 
both carry valine in this position, and the additional van der Waals interactions may give rise to a stronger interaction for these fingers. Similarly, the contact residue S $218_{\text {dFOG }}$ is substituted with arginine in several GATA-binding FOG ZnFs. As shown in Fig. $3 B$, an arginine side chain would be well positioned to make electrostatic interactions with both E200 ${ }_{\mathrm{NF}}$ and E203 ${ }_{\mathrm{NF}}$. Finally, the two residues that are absolutely conserved in all GATA-binding FOG ZnFs, namely $\mathrm{I} 215_{\mathrm{dFOG}}$ and $\mathrm{Y} 230_{\mathrm{dFOG}}$, appear on the periphery of the interface (Fig. 2 Left). Whereas Y $230_{\mathrm{dFOG}}$ makes direct contact with a number of residues in NF, I $215_{\mathrm{dFOG}}$ does not appear to directly contact NF. However, mutation of this residue to alanine abrogates the GATA/FOG interaction. It is possible that $\mathrm{I} 215_{\mathrm{dFOG}}$ assists in establishing the nature of the GATA-binding surface of FOG by excluding solvent and modifying the electrostatics of residues at the interface. This residue also packs against $\mathrm{Y} 230_{\mathrm{dFOG}}$, and might aid in positioning the $\mathrm{Y}_{2} 30_{\mathrm{dFOG}}$ side chain.

Binding Properties of the GATA-1 NF. Although the interaction between GATA-1 and FOG-1 is essential for normal erythropoiesis, the molecular mechanisms underlying this requirement are not well understood. Furthermore, FOG-1 can act as either a corepressor (31) or a coactivator (6) in different assays, and we do not currently understand the basis for this observation. The corepressor activity of FOG could arise from FOG blocking the binding of GATA-1 to DNA, or FOG could serve as a scaffold for the recruitment of additional gene silencing proteins. In contrast, the finding that FOG can work as a coactivator is most consistent with a mechanism where it associates with GATA-1 bound at a gene control element. However, the coassociation of GATA-1 and FOG on a single DNA element has not to our knowledge been demonstrated directly. We used magnetic beads coupled to DNA containing a single GATA element to determine whether murine GATA- 1 can contact both DNA and FOG simultaneously (Fig. 4A). As shown in lane 5, GATA-1 is retained by the wild-type promoter sequence, but binding to this site is abrogated by mutation of the A/TGATAA/G motif (lane 6 ). When FOG is added to the reaction mixture (lane 3 ), it is also retained on the promoter, but only in the presence of GATA-1 (lane 4). Thus, FOG can be recruited to DNA by GATA-1.

We further sought to determine whether the NF can simultaneously contact DNA and FOG. Therefore, we repeated the pull-down by using a GATC DNA element from the ALAS2 promoter, which has been shown to bind the GATA-1 NF (5), and purified recombinant domains from GATA-1 and FOG. In the absence of CF, NF was indeed able to simultaneously bind DNA and FOG finger 6 (Fig. 4B). Notably, the NF/DNA complex selectively bound the full-length FOG construct in preference to truncations that were more abundant in the input, which is in accord with the structure of GATA/FOG complex (Fig. $4 C$, lilac). These data support the view that FOG associates with DNA-bound GATA-1 rather than affecting its DNAbinding activity. The repression observed on some promoters is thus most likely to be due to the recruitment of additional cofactors such as chromatin modifying complexes.

Analysis of GATA-1-Related Human Blood Disorders. At least five distinct $G A T A-1$ mutations that are associated with familial anemias and/or thrombocytopenias have been reported. These mutations all lie in the NF domain and result in single amino acid substitutions: V205M (33), G208S (34), R216Q (35), D218G (36), and D218Y (37). These substitutions give rise to distinct but related genetic disorders. For instance, the V205M and D218Y mutations both give rise to severe anemia and thrombocytopenia, whereas the G208S and D218G mutations give rise to thrombocytopenia but have less significant effects on erythropoiesis. The simplest explanation for these observations is that
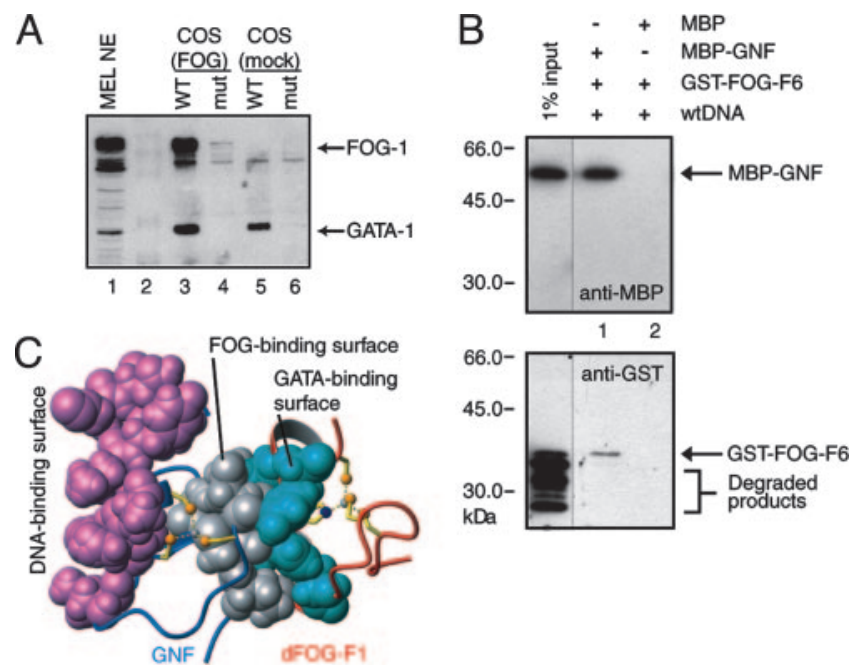

Fig. 4. Simultaneous binding of GATA-1 to DNA and FOG-1. (A) Pull-down showing that full-length GATA-1 can simultaneously bind to the murine $\alpha$-globin promoter and recruit full-length FOG-1. MEL cell nuclear extract (lane 1), supplemented with COS nuclear extract from untransfected [COS(mock); lanes 5 and 6] or transfected [COS(FOG); lanes 3 and 4] cells, was incubated with DNA containing either a wild-type (WT) or a mutant (mut) GATA site coupled to magnetic beads. Complexes were blotted with antibodies against GATA-1 and FOG. (B) Pull-down showing that murine GATA-1 NF can simultaneously bind a GATC site and FOG-1 finger 6. Magnetic beads coupled to a GATC-containing oligonucleotide were incubated with purified MBP-NF and GST-FOG-F6 and run on denaturing gels. Gels were blotted with anti-MBP and anti-GST. A number of breakdown products of GST-FOG-F6 are visible in the input. (C) DNA- and protein-binding surfaces of the GATA/FOG complex. The DNA-binding surface of GATA-1 NF is inferred from the NMR structure of the GATA-1 CF bound to DNA (38). The complex is shown in the same orientation as in Fig. 1D.

the mutations alter the structure and functions of the NF in different ways.

To assess the consequences of each of these mutations, we expressed and purified the mutant NFs and assessed their biophysical properties. Far-UV CD spectra (Fig. $5 A$ ) reveal that all mutants contain substantial levels of secondary structure, but display a blue-shifted minimum $(\approx 5 \mathrm{~nm})$. One-dimensional ${ }^{1} \mathrm{H}$ NMR spectra of these mutants (Fig. 5B) show that only R216Q has a well defined tertiary fold, as judged by the presence of well separated resonances in the range of 9 to $9.5 \mathrm{ppm}$. The combination of $\mathrm{CD}$ and NMR data indicates that the other mutants probably exist in solution as molten-globule-like states that contain substantial amounts of secondary structure but lack a well defined tertiary structure. These ensembles most likely undergo conformational fluctuations on a millisecond to microsecond time scale. Thus, the mutations V205M, G208S, D218G, and D218Y all destabilize the structure of NF, which may contribute to the mechanism by which they impair GATA-1 activity in vivo.

We also determined whether the mutants retained binding functionality. Isothermal titration calorimetry was used to quantitate the ability of each mutant to bind to either DNA or dFOG-F1 (Fig. 5C and Table 1). No measurements were made with D218Y, because of difficulties in obtaining pure, stable protein. V205M, G208S, and D218G were all able to bind to a 16-bp oligonucleotide containing a GATC site with similar affinities to wild-type NF. $\mathrm{R} 216 \mathrm{Q}$ showed no measurable binding to DNA. We then tested the ability of the domains to interact with dFOG-F1. R216Q and D218G bound to dFOG-F1 with affinities that were indistinguishable from that of wild-type GATA-1 NF, whereas V205M and G208S did not bind dFOG-F1. 

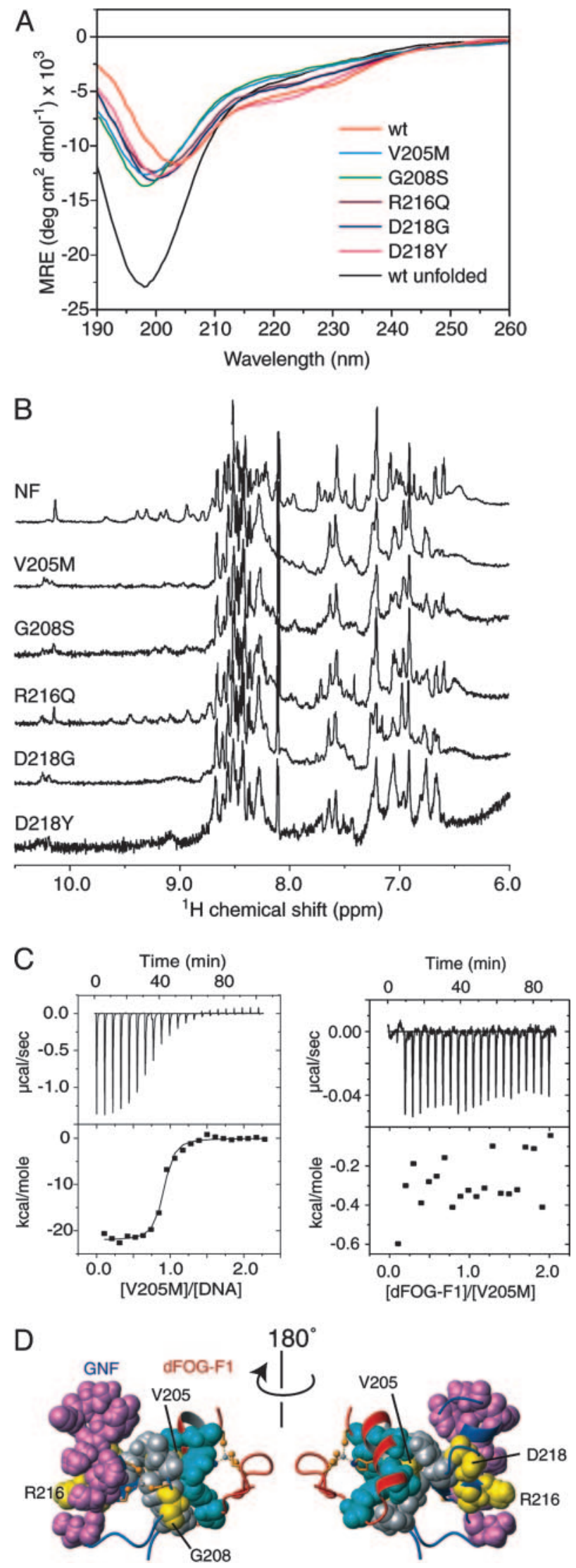

Fig. 5. GATA-1 NF mutations associated with familial blood disorders. $(A)$ Far-UV CD spectra of NF mutants, showing that each forms substantial secondary structure, compared to the wild-type protein in the absence of $\mathrm{Zn}$ (II)
Table 1. DNA- and FOG-binding affinities of naturally occurring GATA-1 mutants

\begin{tabular}{lll} 
& \multicolumn{2}{c}{$k_{\mathrm{a}}$} \\
\cline { 2 - 3 } Protein & \multicolumn{1}{c}{ NF:DNA, M ${ }^{-1}$} & \multicolumn{1}{c}{ NF:FOG, $\mathrm{M}^{-1}$} \\
\hline mNF & $(1.9 \pm 0.1) \times 10^{6}$ & $(4.5 \pm 1.8) \times 10^{4}$ \\
V205M & $(2.0 \pm 1.6) \times 10^{7}$ & No binding \\
G208S & $(9.1 \pm 5.2) \times 10^{6}$ & No binding \\
R216Q & No binding & $(9.4 \pm 0.3) \times 10^{4}$ \\
D218G* & $>10^{7}$ & $(1.9 \pm 0.3) \times 10^{4}$ \\
\hline
\end{tabular}

Association constants were measured by isothermal titration caliometry. Numbers are presented with standard deviations obtained from fitting the data to a simple 1:1 model.

* The sharpness of the binding curve obtained in the D218G/DNA titration prevented accurate determination of the binding affinity, and the affinity is therefore presented as a lower limit.

\section{Discussion}

ZnFs as Protein-Binding Domains. ZnF proteins comprise 3\% of human genes. Although $\mathrm{ZnFs}$ are best recognized as sequencespecific DNA-binding motifs, it has become clear that they also can function as RNA- and protein-recognition motifs. This study provides a demonstration of the molecular mechanisms through which pairs of proteins can interact via their $\mathrm{ZnF}$ domains.

Notably, all of the FOG ZnFs that contact GATA-1 are not part of tandem arrays of ZnFs. Thousands of such "isolated" $\mathrm{ZnFs}$ exist, and it is likely that many serve as protein recognition motifs. The surface used by FOG ZnFs to recognize GATA-1 overlaps with the surface normally used by classical $\mathrm{ZnFs}$ to bind to DNA, indicating that the classical $\mathrm{ZnF}$ has acted throughout evolution as a versatile structural scaffold, onto which different binding functions have been "grafted." In line with this idea, we recently demonstrated that the third classical $\mathrm{ZnF}$ from FOG mediates a specific interaction with the coiled-coil protein TACC3 (9). Indeed, given that a single classical $\mathrm{ZnF}$ is capable of mediating protein-protein interactions, and that an array of such domains is necessary for high affinity DNA binding, it is likely that the latter function arose later as a consequence of gene duplication events.

GATA-1-Related Blood Disorders. The structure of the GATA/FOG complex, together with our biophysical data, provides insights into the molecular basis for hematological disorders that arise from inherited mutations in GATA-1. Fig. $5 D$ shows the location of each of the mutations on NF. It is immediately apparent that the FOG- and DNA-binding surfaces of NF are nonoverlapping, consistent with our finding that NF can participate in both interactions simultaneously.

R216 clearly lies on the DNA-binding surface of NF; the corresponding residue forms an ionic interaction with the phosphate backbone in the structure of the CF/DNA complex (38). Mutation to glutamine will remove this interaction. Our isothermal titration calorimetry data reveal that the R216Q mutant does not bind DNA. Despite this, CD and NMR data indicate that this mutant is well folded, and is also still able to bind

(black). (B) One-dimensional ${ }^{1} \mathrm{H}$ NMR spectra of the proteins in A. V205M, G208S, D218G, and D218Y do not form compact tertiary structures in the same way as the wild-type or R216Q. (C) Isothermal titration calorimetry data showing the titration of dFOG-F1 into V205M (Right) and the titration of V205M into a 16-bp olignonucleotide (Left). (D) Locations of the NF mutations (yellow) in the GATA/FOG complex. The complex is shown in the same orientation as in Fig. 1. The DNA-binding surface of NF is shown in purple, whereas the FOG-binding face is shown in gray. R216 lies on the NF/DNA interface, V205 and G208 lie on the FOG contact surface, and D218 lies between these two opposing surfaces. 
dFOG-F1 with wild-type affinity. This latter observation is not surprising, given the relative locations of the FOG- and DNAbinding sites. Thus, the only mechanism that we have detected through which $\mathrm{R} 216 \mathrm{Q}$ causes disease is the abrogation of DNA-binding by the NF. The NF is thought to play a relatively minor role in DNA-binding in vivo, and the relatively mild phenotype of individuals carrying the R216Q mutation is consistent with this view.

The mutation V205M lies at the center of the FOG-binding surface of NF. As shown in Fig. 2, the V205 side chain fits snugly into a cavity on the surface of the FOG finger and is completely buried in the complex. Methionine cannot occupy the same cavity, and the fact that we observed no FOG binding with this mutant confirms the importance of V205 in the GATA/FOG interaction. This mutation causes severe anemia and thrombocytopaenia, suggesting that abrogation of the GATA-1/FOG interaction profoundly impairs both erythropoiesis and thrombopoiesis. This conclusion is in accord with the phenotype observed for FOG-knockout mice (7).

Interestingly, this mutant retains full DNA-binding ability, despite the fact that its ${ }^{1} \mathrm{H}$ NMR spectrum indicates reduce levels of tertiary structure. This observation is in agreement with the findings of Crispino et al. (8), which showed that the mutation V205G (which removes the hydrophobic valine side chain) disrupted the interaction of NF with FOG but not with DNA. We have recently made a similar observation for the binding of the zinc-finger transcriptional regulator basic Krüppel-like factor (39) to DNA: partially folded BKLF mutants retained full DNA-binding capacity. These data demonstrate that mild destabilization of a protein fold, even to the extent of disrupting the tertiary structure, does not necessarily compromise function. Changes in the enthalpic and entropic contributions to binding often dampen effects on the free energy change, a phenomenon referred to as enthalpy-entropy compensation. This situation presumably minimizes the impact of random mutations on an organism.

1. Weiss, M. J. \& Orkin, S. H. (1995) Exp. Hematol. 23, 99-107.

2. Cantor, A. B. \& Orkin, S. H. (2002) Oncogene 21, 3368-3376.

3. Krishna, S. S., Majumdar, I. \& Grishin, N. V. (2003) Nucleic Acids Res. 31, 532-550.

4. Trainor, C. D., Ghirlando, R. \& Simpson, M. A. (2000) J. Biol. Chem. 275, 28157-28166.

5. Newton, A., Mackay, J. \& Crossley, M. (2001) J. Biol. Chem. 276, 35794-35801.

6. Tsang, A., Visvader, J. E., Turner, C. A., Fujiwara, Y., Yu, C., Weiss, M. J. \& Orkin, S. (1997) Cell 90, 109-119.

7. Tsang, A. P., Fujiwara, Y., Hom, D. B. \& Orkin, S. H. (1998) Genes Dev. 12, 1176-1188.

8. Crispino, J., Lodish, M. B., Mackay, J. P. \& Orkin, S. H. (1999) Mol. Cell. 3, 219-228.

9. Simpson, R. J. Y., Lee, S. H. Y., Bartle, N., E. Y., S., Visvader, J. E., Matthews, J. M., Mackay, J. P. \& Crossley, M. (2004) J. Biol. Chem. 279, 39789-39797. 10. Mackay, J. P. \& Crossley, M. (1998) Trends Biochem. Sci. 23, 1-4.

11. Liew, C. K., Kowalski, K., Fox, A. H., Newton, A., Sharpe, B. K., Crossley, M. \& Mackay, J. P. (2000) Structure (London) 8, 1157-1166.

12. Bartels, C., Xia, T.-H., Billeter, P., Guntert, P. \& Wüthrich, K. (1995) J. Biomol. NMR 5, 1-10.

13. Clore, G. M. \& Gronenborn, A. M. (1994) Methods Enzymol. 239, 349-363.

14. Wishart, D. S. \& Sykes, B. D. (1994) J. Biomol. NMR 4, 171-180.

15. Bax, A., Vuister, G. W., Grzesiek, S., Delaglio, F., Wang, A. C., Tschudin, R. \& Zhu, G. (1994) Methods Enzymol. 239, 79-105.

16. Cornilescu, G., Delaglio, F. \& Bax, A. (1999) J. Biomol. NMR 13, 289-302.

17. Otting, G. \& Wüthrich, K. (1989) J. Magn. Reson. 85, 586-594.

18. Guntert, P., Mumenthaler, C. \& Wüthrich, K. (1997) J. Mol. Biol. 273, 283-298.

19. Nilges, M. (1995) J. Mol. Biol. 245, 645-660.

20. Nilges, M., Macias, M. J., O’Donoghue, S. I. \& Oschkinat, H. (1997) J. Mol. Biol. 269, 408-422.

21. Kwan, A. H., Gell, D. A., Verger, A., Crossley, M., Matthews, J. M. \& Mackay, J. P. (2003) Structure (London) 11, 803-813.

22. Dominguez, C., Boelens, R. \& Bonvin, A. M. (2003) J. Am. Chem. Soc. 125, 1731-1737.
The G208S mutant, like V205M, is partially folded, unable to bind to dFOG-F1 in vitro, but retains DNA-binding ability. In the NF structure, $\mathrm{G} 208$ takes up a positive $\phi$ angle $\left(\approx 100^{\circ}\right)$, so it is not surprising that mutation of this residue disrupts the structure of NF to some degree. The disruption occurs on the periphery of the FOG-binding surface. Interestingly, the phenotype for patients carrying this mutation is less severe than that of V205M (thrombocytopenia only), and previous gene reporter assays have indicated that the effect of the G208S mutation is less pronounced. In fact, we also showed recently that G208S appears to weakly interact with FOG-F6 (34).

$\mathrm{D} 218 \mathrm{G}$ is perhaps the most intriguing mutation. Although the DNA-binding affinity could not be accurately defined because of the properties of the binding isotherm, it was clear that the mutation does not significantly compromise binding to either DNA or dFOG-F1. D218 is located in the second $\beta$-hairpin of $\mathrm{NF}$ and lies on a surface midway between the DNA- and FOG-binding faces of NF (Fig. 5D). This residue does not contact FOG and is not expected to contact DNA, so how then does mutation of $\mathrm{D} 218$ give rise to thrombocytopaenia and mild dyserythropoiesis? It has been noted that binding of D218G to some FOG fingers (e.g., mammalian FOG-finger 1) is reduced, and this may cause problems. Alternatively, this residue may be important for the recognition of other partners of GATA-1, such as CBP, Fli-1 or PU.1.

Our data show how ZnFs can act as protein recognition modules, and demonstrate how even domains as small as $30-40$ aa can present two (or perhaps more) completely separable functional surfaces. These findings also shed light on the molecular origins of GATA-1-related blood disorders.

This work was supported by Australian Research Council and National Health and Medical Research Council (NHMRC) grants (to J.P.M. and M.C.). J.P.M. is a NHMRC Senior Research Fellow. J.M.M. is a Charles and Sylvia Viertel Medical Research Fellow. C.K.L., R.J.Y.S., A.H.Y.K., and F.E.L. are recipients of Australian Postgraduate Awards. L.A.C. is a NHMRC C. J. Martin Fellow.

23. Kowalski, K., Czolij, R., King, G. F., Crossley, M. \& Mackay, J. P. (1999) J. Biomol. NMR 13, 249-262.

24. Laskowski, R. A., Rullmannn, J. A., MacArthur, M. W., Kaptein, R. \& Thornton, J. M. (1996) J. Biomol. NMR 8, 477-486.

25. Wallace, A., Laskowski, R. \& Thornton, J. (1995) Prot. Eng. 8, 127-134.

26. Kowalski, K., Liew, C. K., Matthews, J. M., Gell, D. A., Crossley, M. \& Mackay, J. P. (2002) J. Biol. Chem. 277, 35720-35729.

27. Matthews, J. M., Kowalski, K., Liew, C. K., Sharpe, B. K., Fox, A. H., Crossley, M. \& MacKay, J. P. (2000) Eur. J. Biochem. 267, 1030-1038.

28. Haenlin, M., Cubadda, Y., Blondeau, F., Heitzler, P., Lutz, Y., Simpson, P. \& Ramain, P. (1997) Genes Dev. 11, 3096-3108.

29. Fossett, N., Tevosian, S. G., Gajewski, K., Zhang, Q., Orkin, S. H. \& Schulz, R. A. (2001) Proc. Natl. Acad. Sci. USA 98, 7342-7347.

30. Fossett, N., Hyman, K., Gajewski, K., Orkin, S. H. \& Schulz, R. A. (2003) Proc. Natl. Acad. Sci. USA 100, 11451-11456.

31. Fox, A., Kowalski, K., Mackay, J. P. \& Crossley, M. (1999) EMBO J. 18, 2812-2822.

32. Lo Conte, L., Chothia, C. \& Janin, J. (1999) J. Mol. Biol. 285, 2177-2198

33. Nichols, K. E., Crispino, J. D., Poncz, M., White, J. G., Orkin, S. H., Maris, J. M. \& Weiss, M. J. (2000) Nat. Genet. 24, 266-270.

34. Mehaffey, M. G., Newton, A. L., Gandhi, M. J., Crossley, M. \& Drachman, J. G (2001) Blood 98, 2681-2688.

35. Yu, C., Niakan, K. K., Matsushita, M., Stamatoyannopoulos, G., Orkin, S. H. \& Raskind, W. H. (2002) Blood 100, 2040-2045.

36. Freson, K., Devriendt, K., Matthijs, G., Van Hoof, A., De Vos, R., Thys, C., Minner, K., Hoylaerts, M. F., Vermylen, J. \& Van Geet, C. (2001) Blood 98, $85-92$.

37. Freson, K., Matthijs, G., Thys, C., Marien, P., Hoylaerts, M. F., Vermylen, J. \& Van Geet, C. (2002) Hum. Mol. Genet. 11, 147-152.

38. Omichinski, J. G., Clore, G. M., Schaad, O., Felsenfeld, G., Trainor, C., Appella, E., Stahl, S. J. \& Gronenborn, A. M. (1993) Science 261, 438-446.

39. Simpson, R. J. W., Cram, E. D., Czolij, R., Matthews, J. M., Crossley, M. \& Mackay, J. P. (2003) J. Biol. Chem. 278, 28011-28018. 